\title{
Anisotropy of Protons and Light Primary Nuclei in Cosmic Rays Measured with the Alpha Magnetic Spectrometer on the ISS
}

\section{A. Velasco ${ }^{a, *}$ and J. Casaus, C. Mañá, M. Molero ${ }^{a}$}

${ }^{a}$ Centro de Investigaciones Energéticas, Medioambientales y Tecnológicas (CIEMAT), Avda. Complutense 40, Madrid, Spain

E-mail: MiguelAngel.Velasco@Ciemat.es

\begin{abstract}
A measurement of the cosmic ray anisotropy on the arrival directions of protons and light primary nuclei (helium, carbon and oxygen) has been performed in galactic coordinates by the Alpha Magnetic Spectrometer onboard the International Space Station. The analysis is based on the sample of events collected in the first 9 years of data taking. The results for the different particle species are consistent with isotropy and upper limits on the dipole amplitude $(\delta)$ have been computed. In particular, an upper limit of $\delta<0.32 \%$ at the $95 \%$ C.I. is established for galactic protons above $200 \mathrm{GV}$. On the other hand, limits on the dipole anisotropy of galactic helium, carbon and oxygen above $200 \mathrm{GV}$ are quoted: $\delta<0.32 \%, \delta<1.62 \%$ and $\delta<1.69 \%$, respectively.
\end{abstract}

$37^{\text {th }}$ International Cosmic Ray Conference (ICRC 2021)

July 12th-23rd, 2021

Online - Berlin, Germany

\footnotetext{
*Presenter
} 


\section{Introduction}

In the last years, the Alpha Magnetic Spectrometer (AMS-02) onboard the International Space Station (ISS) has provided precise measurements of cosmic ray fluxes, which have revealed many unexpected features that cannot be fully explained within the current understanding of cosmic rays acceleration and propagation.

The measurement of the proton and light primary nuclei fluxes [1] does not follow a single power law and progressively hardens at high rigidities, with an increase of the spectral index above $200 \mathrm{GV}$. Many models have been proposed to explain this spectral feature according to distinct contributions: different acceleration mechanisms at source [2-4], propagation effects [5-7], and local sources $[8,9]$.

The different mechanisms proposed to account for the spectral hardening of protons and light primary nuclei may have a distinct impact in their arrival directions. In particular, the presence of a local source may imprint a sizable anisotropy in their flux at high rigidities. Therefore, the measurement of the directionality of the particle fluxes may provide additional information to understand the origin of these features.

\section{The AMS-02 detector}

AMS-02 is a multipurpose particle physics detector designed to carry out accurate measurements of cosmic ray charged particles in the GeV-TeV range. It was installed on 19 May 2011 onboard the International Space Station and it continues taking data steadily since then. So far, AMS-02 has collected more than 180 billion events of galactic cosmic rays in a long term mission, which is supposed to continue during ISS lifetime until 2028.

The detector consists of nine layers of precision silicon tracker (STD), with an inner tracker (L2-L8) inside a permanent magnet and two outer layers (L1 and L9); a transition radiation detector (TRD); four planes of time of flight counters (TOF); an array of anti-coincidence counters (ACC) surrounding the inner tracker; a ring imaging Čerenkov detector (RICH); and an electromagnetic calorimeter (ECAL). More details on the sub-detectors can be found in [10].

\section{Data selection}

Proton, helium, carbon and oxygen events are required to be downward-going particles with velocity measured by four TOF planes and charge consistent to $Z=1,2,6,8$ respectively.

Events reconstructed by the 4 inner tracker planes, passing through the outer layer L1, and satisfying additional track quality criteria are finally selected. For the proton sample, an additional hit in the outer layer L9 is required.

In addition, to select only primary cosmic rays well above the geomagnetic cutoff, the measured rigidity is required to be greater than the maximum geomagnetic cutoff within the AMS field of view.

Above $18 \mathrm{GV}$, the samples used in the analysis of anisotropies for the first 9 years of AMS include $1.4 \times 10^{8}$ protons, and $1.2 \times 10^{8}, 3.8 \times 10^{6}$ and $3.5 \times 10^{6}$ helium, carbon and oxygen events, respectively. The total exposure time of this analysis amounts to $1.91 \times 10^{8} \mathrm{~s}$. 
The anisotropy analysis is performed on 9 cumulative rigidity ranges of minimum rigidity: 18 , $30,45,80,150,200,300,500,1000 \mathrm{GV}$.

\section{Methodology}

The analysis of anisotropies in a sample is performed by comparing the observed distribution of arrival directions in galactic coordinates, $(l, b)$, with a reference map. Skymaps for both sample of study and reference are built using HEALPix scheme [11], which ensures a pixelization with an equal area isolatitude subdivisions of the sphere.

The reference map for anisotropy studies describes the directional response of the detector to an isotropic flux, and its computation requires a detailed understanding of experimental effects. In particular, a geographical variation of detector efficiencies may project onto the galactic coordinate system and induce an spurious signal if not accounted. Therefore, a precise knowledge of these variations is needed to disentangle a possible physical signal from the effects related to the performance of the detector. A systematic procedure, valid for all cosmic ray species, has been developed to obtain these isotropic skymaps [12].

A likelihood fit is used to compare the distribution of events under study and the reference map, and takes into account the differences in the exposure for different energies or rigidities due to geomagnetic cutoff variation along the orbit. This procedure proved to be stable against different map resolutions and sample statistics. A spherical harmonics expansion in terms of multipolar coefficients, $a_{\ell m}$, is used to described the directional dependence of the flux:

$$
\Phi(l, b)=\Phi_{0}\left(1+\sum_{\ell>0} \sum_{m=-\ell}^{m=+\ell} a_{\ell m} Y_{\ell m}(l, b)\right)
$$

At first order, $\ell=1$, the dipole is fully described by three orthonormal functions corresponding to three orthogonal axes: $Y_{1+1}$ is aligned with the Forward-Backward direction, pointing to the galactic center; $Y_{1+0}$ is aligned with the North-South direction, pointing to the north galactic pole; and $Y_{1-1}$ is aligned with the East-West direction, contained in the galactic plane and completes the right-handed coordinate system. Dipole components in each direction are defined as

$$
\rho_{\mathrm{EW}}=\sqrt{\frac{3}{4 \pi}} a_{1-1} \quad ; \quad \rho_{\mathrm{NS}}=\sqrt{\frac{3}{4 \pi}} a_{1+0} \quad ; \quad \rho_{\mathrm{FB}}=\sqrt{\frac{3}{4 \pi}} a_{1+1}
$$

Finally, the dipole amplitude, which quantifies the asymmetry between the maximum and minimum of the flux, is

$$
\delta=\frac{\Phi_{\max }-\Phi_{\min }}{\Phi_{\max }+\Phi_{\min }}=\sqrt{\rho_{\mathrm{EW}}^{2}+\rho_{\mathrm{NS}}^{2}+\rho_{\mathrm{FB}}^{2}}
$$

\section{Proton anisotropy}

The results of the measurement of the proton anisotropy in galactic coordinates are presented in figure 1 . The proton anisotropy is dominated by statistics above $70 \mathrm{GV}$ and systematics limit the measurement at low rigidities to $0.1 \%$. The values of the dipole components are consistent with isotropy for the different rigidity ranges of the analysis. Consequently, upper limits on the proton dipole amplitude are computed at the $95 \%$ credible interval (C.I.). In particular, for rigidities above $200 \mathrm{GV}$, the upper limit is set to $\delta<0.32 \%$. 

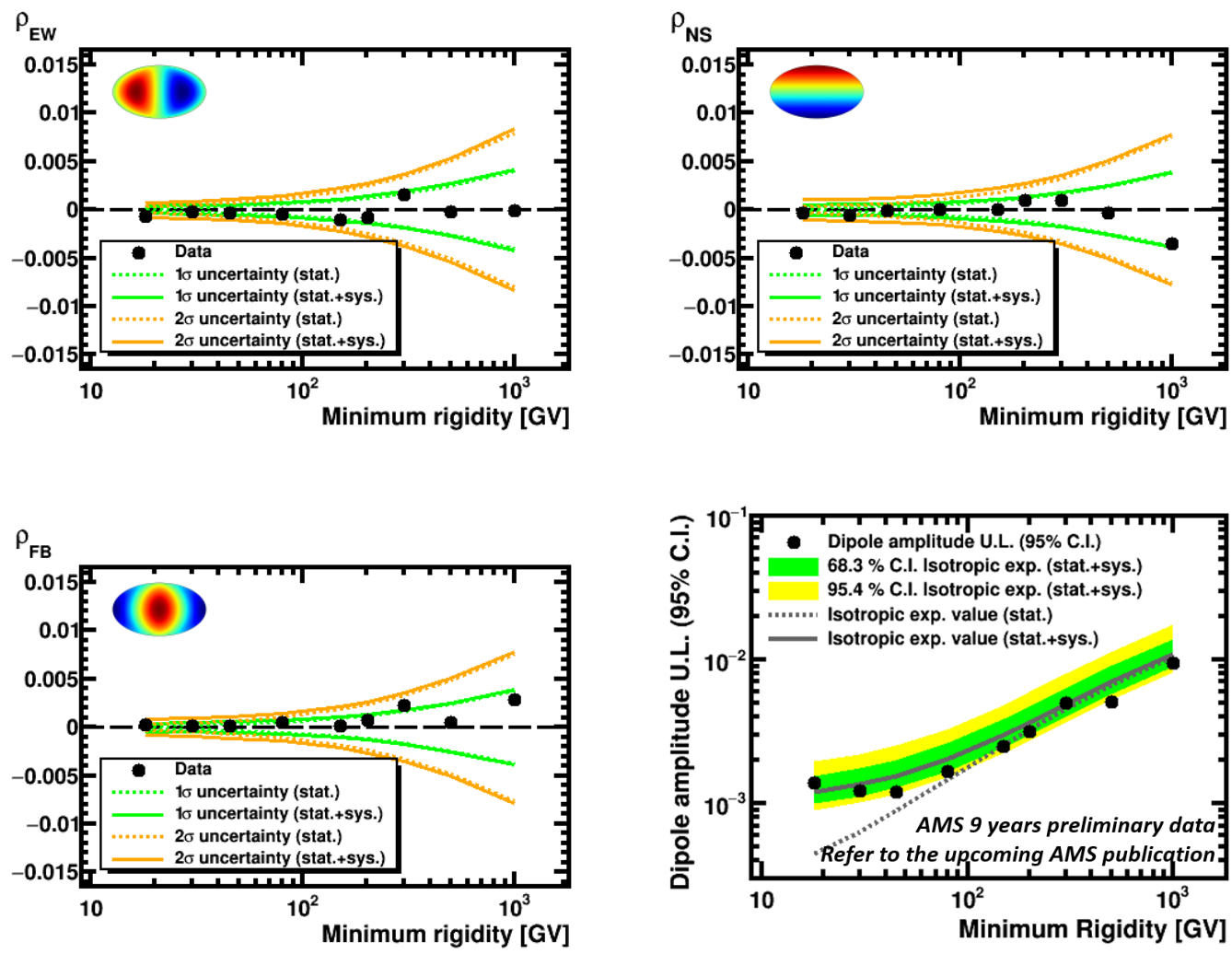

Figure 1: Dipole components of the proton anisotropy in galactic coordinates: East-West (top left), NorthSouth (top right) and Forward-Backward (bottom left) as a function of the minimum rigidity. 95\% C.I. upper limits on the dipole amplitude (bottom right). Bands corresponding to the isotropic expectation at $68.3 \%$ (green) and $95.4 \%$ C.I. (yellow) are also displayed.

\section{Light primary nuclei anisotropy}

The study of the helium anisotropy provides the dipole components presented in figure 2 . The analysis is dominated by statistics in the entire rigidity range and the results are consistent with isotropy. Therefore, upper limits to the helium dipole amplitude are calculated for each rigidity range. In particular, above $200 \mathrm{GV}$ the helium dipole anisotropy is found to be $\delta<0.32 \%$ at the $95 \%$ C.I.

A similar analysis is performed on the sample of carbon and oxygen events. In both cases, the results are consistent with isotropy and upper limits on the dipole amplitude are computed (figure 3). Above $200 \mathrm{GV}$, the upper limits are established to $\delta<1.62 \%$ for carbon and $\delta<1.69 \%$ for oxygen at the $95 \%$ C.I. 
$\rho_{\text {EW }}$

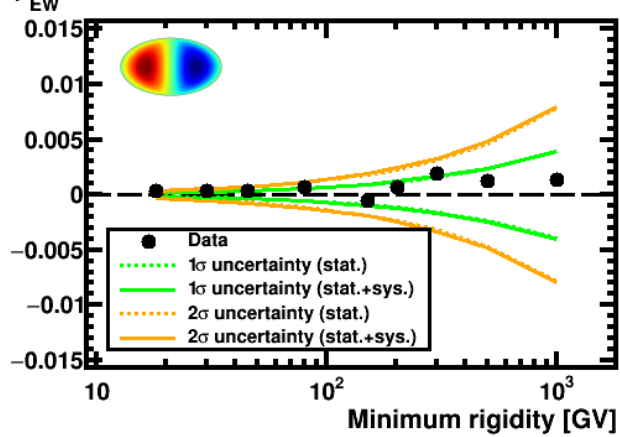

$\rho_{\text {FB }}$

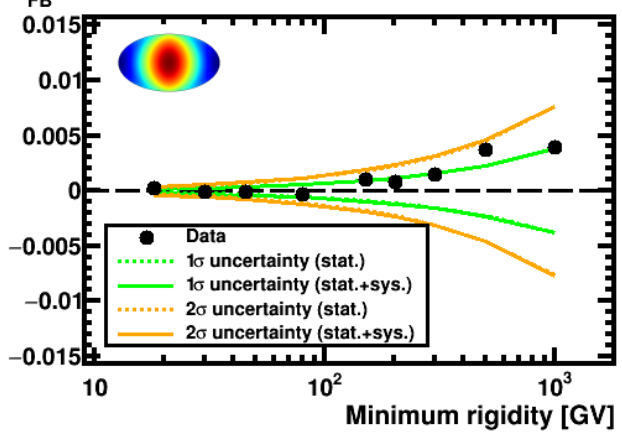

$\rho_{\mathrm{NS}}$
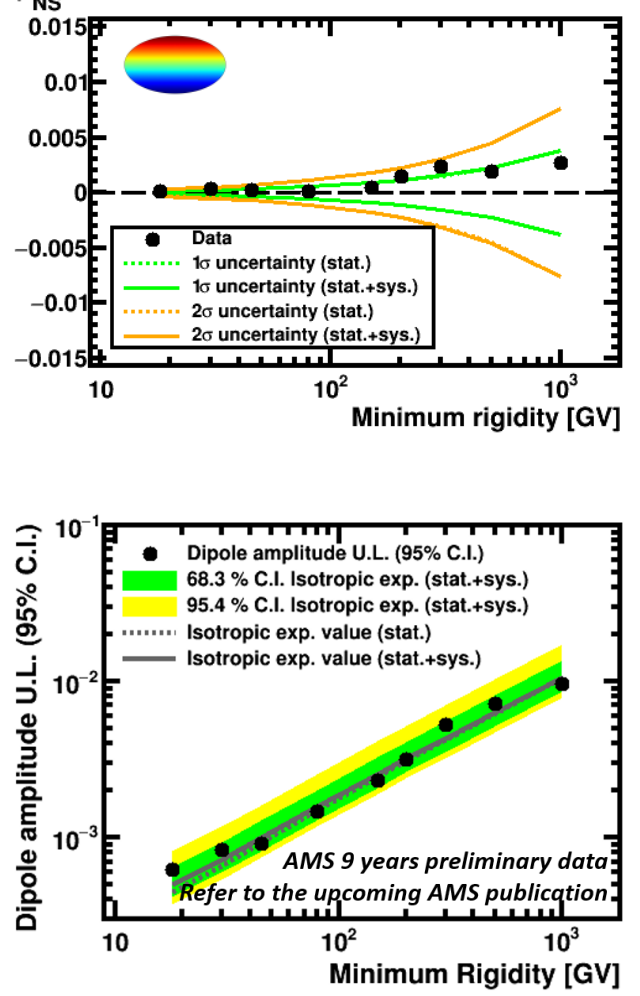

Figure 2: Dipole components of the helium anisotropy in galactic coordinates: East-West (top left), NorthSouth (top right) and Forward-Backward (bottom left) as a function of the minimum rigidity. 95\% C.I. upper limits on the dipole amplitude (bottom right). Bands corresponding to the isotropic expectation at $68.3 \%$ (green) and $95.4 \%$ C.I. (yellow) are also displayed.
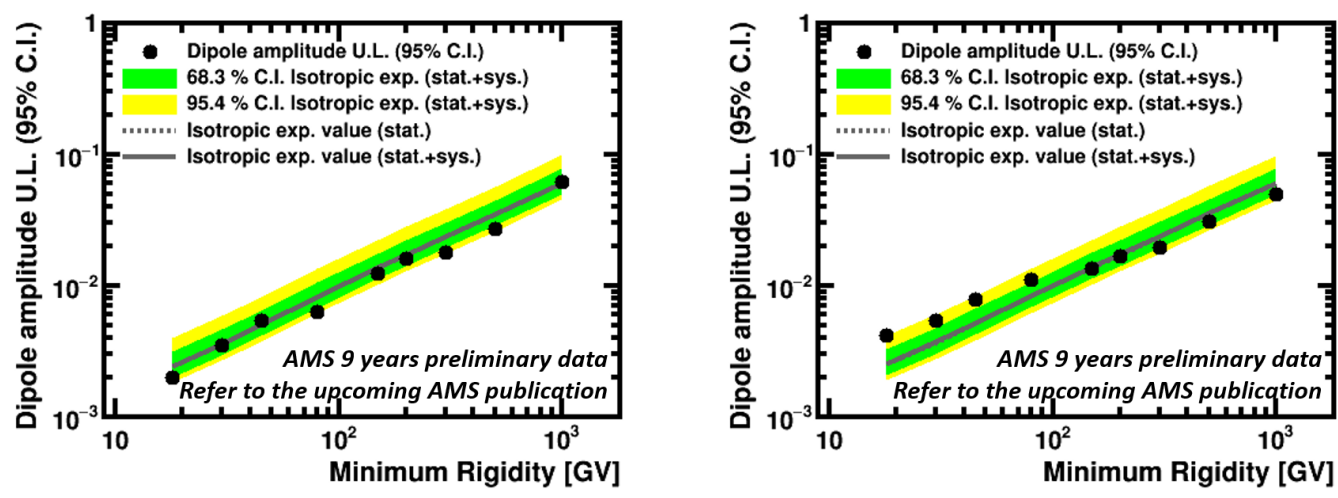

Figure 3: $95 \%$ C.I. upper limits on the dipole amplitude of carbon (left) and oxygen (right). The bands corresponding to the isotropic expectation at $68.3 \%$ (green) and $95.4 \%$ C.I. (yellow) are also displayed. 


\section{Conclusions}

The measurement of the anisotropy in the arrival directions of galactic cosmic ray protons and light primary nuclei may provide additional information to understand the origin of the features observed in their spectra.

A measurement of the anisotropy of protons and light primary nuclei (helium, carbon and oxygen) in galactic coordinates has been carried out by the AMS-02 experiment on the ISS using 9 years of data. The results obtained for these particle species show no deviation from isotropy and $95 \%$ C.I. upper limits on the dipole amplitude have been established. In particular, the proton dipole amplitude is $\delta<0.32 \%$ above $200 \mathrm{GV}$. On the other hand, upper limits of $\delta<0.32 \%, \delta<1.62 \%$ and $\delta<1.69 \%$ for helium, carbon and oxygen, respectively, are obtained above $200 \mathrm{GeV}$.

\section{References}

[1] M. Aguilar et al. (AMS Collaboration), The Alpha Magnetic Spectrometer (AMS) on the International Space Station: Part II - Results from the First Seven Years, Phys. Rep. 8941 (2021)

[2] W.-H. Lin, B.-W. Bao, Z.-J. Jiang, L. Zhang, Spectral hardening of cosmic ray protons and helium nuclei in supernova remnant shocks, Chinese Phys. C 43053103 (2019)

[3] Y. Zhang, S. Liu, Q. Yuan, Anomalous Distributions of Primary Cosmic Rays as Evidence for Time-dependent Particle Acceleration in Supernova Remnants, ApJ , 844:L3 (2017) [astro-ph.HE/1707.00262]

[4] V. Ptuskin, V Zirakashvili, E.-S. Seo, Spectra of Cosmic-Ray Protons and Helium Produced in Supernova Remnants, ApJ 763(1):47 (2013) [astro-ph.HE/1212 .0381]

[5] Y.-Q. Guo, Z. Tian, C. Jin, Spatial-Dependent Propagation of Cosmic Rays Results in Spectrum of Proton, Ratios of $\bar{p} / p, B / C$ and Anisotropy of Nuclei, ApJ 819:54 (2016) [astro-ph.HE/1509.08227]

[6] N. Tomassetti, Origin of the Cosmic-Ray Spectral Hardening, ApJ 752:L13 (2012) [astro-ph.HE/1204.4492]

[7] P. Blasi, E. Amato, and P. D. Serpico, Spectral Breaks as a Signature of Cosmic Ray Induced Turbulence in the Galaxy, Phys. Rev. Lett. 109, 061101 [astro-ph.HE/1207. 3706]

[8] W. Liu, X.-J. Bi, S.-J. Lin, B.-B. Wang, P.-F. Yin, Excesses of cosmic ray spectra from a single nearby source, Phys. Rev. D 96, 023006 (2017) [astro-ph.HE/1611.09118]

[9] G. Bernard, T. Delahaye, Y.-Y. Keum, W. Liu, P. Salati, and R. Taillet, TeV cosmic-ray proton and helium spectra in the myriad model, A\&A 555, A48 (2013) [astro-ph.HE/1207.4670]

[10] M. Aguilar et al. (AMS Collaboration), First Result from the Alpha Magnetic Spectrometer on the International Space Station: Precision Measurement of the Positron Fraction in Primary Cosmic Rays of 0.5-350 GeV, Phys. Rev. Lett. 110141102 (2013) 
[11] K. M. Górski et al., HEALPix: A Framework for High-Resolution Discretization and Fast Analysis of Data Distributed on the Sphere, ApJ 622759 (2005) [astro-ph. HE/0409513]

[12] M.A. Velasco, Ph. D. Thesis, Universidad Complutense de Madrid, 2018;

M. Molero, Ph. D. Thesis, Universidad Autónoma de Madrid, 2021 